\title{
Special Issue on Stringology
}

\author{
Preface
}

Strings, sequences of symbols, are one of the most natural and widespread representations of information and the study of algorithms, data structures and properties of strings pervades computer science. Everyday examples of the impact of these studies abound: from search engines and the World Wide Web, to marvels in computational biology such as the Human Genome Project, efficient string processing has a profound effect on all of humanity.

The articles collected in this special issue have arisen from the first two installments of StringMasters a meeting of stringology researchers hosted by the Department of Computing and Software, at McMaster University in July 2007 and May 2008. Relaxed in atmosphere, the meetings served as collegiate forums in which professors and graduate students alike discussed various open problems of the field. Much progress was made, and since, several works have arisen from the discussions, a subset of which we present here. In line with the standards of Fundamenta Informaticae, all papers underwent two rounds of reviewing by at least three reviewers per paper.

In the paper Combinatorics of Unique Maximal Factorization Families (UMFFs), Daykin, Daykin and Smyth prove several intricate combinatorial results which relate to an old and deep concept in stringology, Lyndon words.

The article Faster Algorithms for Computing Maximal Multirepeats in Multiple Sequences by Iliopoulos, Smyth and Yusufu describes an algorithm for the efficient discovery of repetitive structures across multiple strings. This problem is of particular significance to data mining in biological data, where the strings may be protein sequences, and the discovered repetitions seed for motifs.

Travis Gagie in A New Algorithm for Building Alphabetic Minimax Trees presents an elegant method for the construction of lexicographic prefix codes given only a sample of symbols from a string requiring compression.

Finally, in Toward a General Framework for Polyphonic Comparison, Allali, Ferraro, Hanna, Iliopoulos and Robine explored the possibilities of extending the edit-distance operations introduced by Mongeau and Sankoff in order to establish a general framework for polyphonic music comparison. And, with this final article we close the special issue.

We warmly thank all the anonymous reviewers for timely and thorough reviewing of the articles, which made our job as editors so much easier. Thanks must also go to the authors for their patience and 
timely revision during the multiple cycle of reviews. Finally, we would also like to thank the Editors of Fundamenta Informaticae for giving us the opportunity to present this snapshot of Stringology to a wider audience.

\title{
The Guest Editors
}

Ryszard Janicki

Department of Computing and Software

McMaster University

Hamilton, Ontario, Canada L8S 4K1

janicki@cas.mcmaster.ca
Simon J. Puglisi

School of Computer Science and Information Technology

Royal Melbourne Institute of Technology

Melbourne, Australia

simon.puglisi@rmit.edu.au

\author{
M. Sohel Raman \\ Dhaka, Bangladesh \\ and \\ Department of Computer Science \\ Kings College London \\ London, UK \\ msrahman@cse.buet.ac.bd
}

Department of Computer Science and Engineering

Bangladesh University of Engineering and Technology 